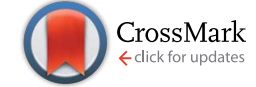

Cite this: RSC Adv., 2017, 7, 5297

Received 18th December 2016 Accepted 26th December 2016

DOI: $10.1039 / \mathrm{c} 6 \mathrm{ra} 28364 \mathrm{k}$

www.rsc.org/advances

\section{Averaging effect on improving signal reproducibility of gap-based and gap-free SERS substrates based on ordered Si nanowire arrays $\uparrow$}

\begin{abstract}
Jian-An Huang, \$Y Yingqi Zhao, $\$$ Xiaoyue Zhu and Wenjun Zhang*
While the surface-enhanced Raman spectroscopy (SERS) technique has the merit of an inherent ultrahigh sensitivity, low signal reproducibility due to nonuniformities in SERS substrates is a challenge to be considered for its practical applications. Utilizing averaging effects to improve the signal reproducibility can be achieved by either increasing hot spot density on the SERS substrates or using large laser spots to enlarge the probing area. However, mechanisms of how the averaging effects overcome the nonuniformities and improve the reproducibility remain unclear. Herein, we use ordered SERS substrates with designed gap-based and gap-free electric fields in the hot spots to systematically study the mechanisms. Our result suggests that the highest reproducibility comes from the gap-free hot spots that depend on the least parameters. In contrast to the gap-based substrate with a trade-off relation between reproducibility and enhancement, the gap-free substrate exhibits both high reproducibility and high enhancement. Our findings clarify the contributions of the SERS substrate to the averaging effect and could pave the way to the design of reproducible SERS substrates for further practical applications.
\end{abstract}

\section{Introduction}

Surface enhanced Raman spectroscopy (SERS), as a fast, highly selective and information-rich detection technique, has been widely used in the field of chemical and biological analysis. ${ }^{1-10}$ Development of SERS systems used to focus on achieving high enhancement factors and low detection limitation. ${ }^{11-13}$ Various hot spot fabrication strategies have been demonstrated for SERS, from random aggregation of noble metal nanoparticles to ordered structures fixed on solid state substrates. ${ }^{14,15}$ Conventional gap-based SERS substrates such as silver nanoparticles distributed on a silicon wafer exhibit very high SERS enhancement that depends on hot spots formed by adjacent nanoparticle pairs, but they usually suffer from low signal reproducibility. ${ }^{15-17}$ The high SERS enhancement of the gapbased substrates is due to coupling of enhanced electric field of the adjacent nanoparticles in $<10 \mathrm{~nm}$ inter-particle gap that depend on the nanoparticle size, inter-particle gap distance and molecule distribution inside the gap. ${ }^{18-20}$ Therefore, any nonuniformity of the three parameters will lead to field variations and resultant fluctuating SERS signals. Signal reliability

\footnotetext{
Center of Super-Diamond and Advanced Films (COSDAF), Department of Physics and Materials Science, City University of Hong Kong, Hong Kong SAR. E-mail: apwjzh@ cityu.edu.hk

$\dagger$ Electronic supplementary information (ESI) available: Enhancement factor calculations, additional information, figures, and references. See DOI: $10.1039 / \mathrm{c} 6 \mathrm{ra} 28364 \mathrm{k}$

\$ These authors contributed equally to this work.
}

and reproducibility in detection are important factors to be concerned for practical applications, especially in the quantitative detections and multiplexing component detections, because fluctuating signals hinder the evaluation of the molecule type and amount. ${ }^{14,21}$ For example, in using the gap-based SERS substrate to image cell membrane dynamics with time, it was difficult to differentiate whether the signal changes was due to the substrate or to membrane lipids..$^{22,23}$

In order to generate reproducible SERS spectra at any positions of a gap-based SERS substrate, in general, three scales of uniformity in the substrate should be achieved: (1) uniform distribution of enhancing nanostructures over the SERS substrate, (2) uniform distribution of the hot spot over all enhancing nanostructures and (3) uniform distribution of analyte molecules over all hot spots. An enhancing nanostructure can be a metal nanocolloid, a metal nanoparticle dimer, or a metal nanotip that can be used for the SERS applications. When the metal nanocolloids are dropped and then dried on a silicon wafer, they will distribute randomly on the wafer depending on solution drying dynamics. The uniform distribution of the enhancing nanostructures requires that the number of the enhancing nanostructures under an excitation spot should remain the same in different positions over the SERS substrate, which could be achieved in SERS systems based on ordered plasmonic structures by top-down fabrications, such as nanosphere lithography-fabricated plasmonic nanosphere array. $^{24}$

In addition, a hot spot can be any enhancing nanostructures as mentioned that exhibit enhanced electric field at surface 
plasmon resonance for molecule excitation. ${ }^{\mathbf{1 5 , 2 5 , 2 6}}$ The field intensity characterizes how "hot" a hot spot can be and depends on the morphology of the enhancing nanostructures, such as size, shape and inter-particle spacing of the metal nanoparticle dimer. The uniform distribution of the hot spot or the field over all metal nanoparticle dimers therefore requires all dimers on the SERS substrate have similar size, shape and the interparticle spacing. One problem with the gap-based SERS substrates is the strong dependence of electric field on the interparticle spacing $(<10 \mathrm{~nm})$, yet the fabrication of dimer-based substrates with highly precise control of the gap size less than $10 \mathrm{~nm}$ is very challenging. ${ }^{27,28}$

As the final piece of the puzzle, the hot spots cannot generate SERS signals without being occupied by analyte molecules. The even distribution of molecules requires that the number of molecules and their positions in the hot spot remain the same throughout the SERS substrate. However, even on the highlyordered plasmonic nanosphere array with precisely controlled gap size, the homogeneous adsorptions of molecules in the gaps are inherently difficult to achieve. In particular, the high SERS enhancement requires small gap distance, but the narrow gaps make molecules extremely difficult to distribute uniformly on the gaps. ${ }^{18}$

Averaging effect, a common way to improve signal reproducibility in SERS, is based on collecting more SERS signals from either more hot spots or more molecules in one Raman measurement. It can be achieved by modifying either the SERS substrates or Raman measurement settings. For example, in the colloidal-based SERS systems, signal reproducibility can be improved by prolonging signal acquisition time or allowing flowing colloids to enter the collection volume of the Raman measurement objective. ${ }^{29,30}$ On solid-state SERS substrates, the averaging effect can be achieved by enlarging excitation laser spots through changing different measurement objectives to cover more hot spots. ${ }^{31}$ However, excitation of more hot spots might include more array defects. Furthermore, large laser spot must be obtained by a low-magnification objective which corresponds to low signal-collection efficiency. Although the averaging effect could improve the reproducibility of many gapbased SERS substrates, its relation to the electric fields of the enhancing structures and connection to the three scales of uniformity remain unclear. The vague understanding of the averaging effect actually hampers the development of reproducible SERS systems.

In this paper, we report a systematical investigation of two approaches leading to the averaging effects on solid-state SERS substrates: increasing hot spot density on the SERS substrates or using large laser spots to enlarge probing area. The connection between the averaging effects and the three scales of uniformity were analyzed by studying the SERS reproducibility of three ordered SERS substrates: two gap-based substrates including Ag nanoparticles coated on Si nanowire array (AgNP/ SiNW) and continuous $\mathrm{Ag}$ film coated on hexagonal-closepacked nanospheres (AgFON), and one gap-free substrate comprising continuous Ag film coated on Si nanowire array (Ag/ SiNW). The electric fields of the AgNP/SiNWs depend on particle size and inter-particle spacing. Together with particle size and inter-particle spacing, the electric field of the AgFON also depends on the nanosphere array defects. In contrary to these gap-based substrates, the gap-free $\mathrm{Ag} / \mathrm{SiNWs}$ exhibit electric fields that depend only on nanowire size rather than the interparticle gaps. ${ }^{32,33}$ By the designed electric fields of these SERS substrates, we are able to study how averaging effect can take effect through the electric fields to improve the reproducibility. The influence of molecular distribution, molecule type and laser beam size on the averaging effect and reproducibility are also discussed. The results are useful to discriminate contributions of the substrate to the SERS reproducibility and pave a way to the design and optimization of SERS substrates for further practical applications.

\section{Experimental}

\section{Reagents and materials}

Rhodamine 6G (R6G, Sigma-Aldrich 252433), and 4-aminothiophenol (4-ABT, Sigma-Aldrich 422967) were purchased from Sigma-Aldrich Co., LLC. Polystyrene (PS) nanospheres of $300 \mathrm{~nm}$ and $470 \mathrm{~nm}$ in diameter were purchased from Bangs Laboratories, Inc. and Microparticles $\mathrm{GmbH}$, respectively.

\section{Fabrication of $\mathrm{Ag} / \mathrm{SiNWs}$ and $\mathrm{AgNP} / \mathrm{SiNWs}$}

SiNW arrays were constructed on (111) p-type silicon wafers with resistivity of 5-20 $\Omega \mathrm{cm}^{-2}$, and nanosphere lithography was carried out to pattern the SiNWs, as described in previous work. $^{34,35}$ Briefly, $300 \mathrm{~nm}$ PS nanospheres were diluted 1:1 (volume) with ethanol and injected slowly onto water surface to form a monolayer. Then, the monolayer was transferred to a clean silicon wafer by lifting up the Si wafer that was placed previously in the water and drying it in the atmosphere. $\mathrm{O}_{2}$ plasma etching was carried out in the Plasma-Therm 790 RIE system to reduce the PS diameter to $110 \mathrm{~nm}$. During etching, the $\mathrm{O}_{2}$ flow rate was $40 \mathrm{sccm}$, pressure $37 \mathrm{mTorr}$, radio frequency power $30 \mathrm{~W}$ and duration 5 minutes. Then a $20 \mathrm{~nm}$-thick gold layer was coated by electron beam evaporation. After removing PS in chloroform, metal-assisted chemical etching was performed to form a SiNW array by placing the as-prepared substrate in to a mixed etchant solution of hydrofluoric acid and hydrogen peroxide for a few minutes depending on the wire length. ${ }^{35,36}$ For $\mathrm{Ag} / \mathrm{SiNWs}$, a $\sim 40$ nm-thick continuous silver layer was deposited on the SiNWs by high-vacuum magnetron sputtering at a pressure of $10^{-5} \mathrm{mBar}$. AgNP/SiNWs were fabricated by decorating AgNPs on SiNW in a low-vacuum sputtering coater at a pressure $\sim 10^{-3} \mathrm{mBar}$. The low vacuum leads to large-sized $\mathrm{Ag}$ particles sputtered by the plasma. The deposition time was adjusted from $30 \mathrm{~s}, 90 \mathrm{~s}$ to $180 \mathrm{~s}$ to fabricate high-density AgNP/SiNWs samples. Low-density AgNP/SiNWs sample that decorated large AgNP on SiNWs was fabricated by annealing the AgNPs on SiNW samples at $400{ }^{\circ} \mathrm{C}$ for $30 \mathrm{~min}$ under nitrogen atmosphere at a pressure of 1 Bar.

\section{Fabrication of AgFON}

Silver film on nanospheres was fabricated on a silicon wafer. Firstly, $470 \mathrm{~nm}$ PS nanospheres mixed with ethanol (by $1: 1$ 
volume) were injected slowly onto water surface to form a monolayer. By lifting up the Si wafer from the water the monolayer was transferred to a clean silicon wafer, and dried in the atmosphere. Then the wafer was loaded in the thermal evaporation chamber. A silver film of $\sim 40 \mathrm{~nm}$ was evaporated on the top of the nanosphere array to form an AgFON structure.

\section{Raman spectra acquisition}

To form a 4-ABT layer on Ag surface, the substrate was soaked for 8 hours in $10^{-4} \mathrm{M}$ 4-ABT, then washed with ethanol and deionized water to remove excessive molecules, and dried with a nitrogen flow. For Raman measurements of R6G, $2 \mu \mathrm{L}$ analyte solutions were dropted on the substrate, dried in atmosphere and measured under a RENISHAW InVia Raman spectrometer with a HeNe laser (17 $\mathrm{mW}$ and $632.8 \mathrm{~nm})$. The full laser power was $4.4 \mathrm{~mW}$ under a $20 \times$ objective. The exposure time of all mapping processed was $1 \mathrm{~s}$ for one point. Each mapping measurement covered 1000 points or more. The step lengths were $3 \mu \mathrm{m}, 2 \mu \mathrm{m}$, and $1 \mu \mathrm{m}$ under $20 \times, 50 \times$, and $100 \times$ objectives. The numerical apertures of the $20 \times, 50 \times$ and $100 \times$ objectives are $0.4,0.75$ and 0.9 , respectively, which correspond to spot sizes of $3 \mu \mathrm{m}, 1.5 \mu \mathrm{m}$ and $1 \mu \mathrm{m}$, respectively.

\section{Results and discussion}

Fig. 1 shows the scanning electron microscope (SEM) images of the samples used in the evaluation of averaging effects: lowdensity AgNP/SiNWs in Fig. 1a, high-density AgNP/SiNWs in Fig. 1b, Ag/SiNWs in Fig. 1c, and AgFON in Fig. 1d, respectively. The nonuniform AgNPs of low-density AgNP/SiNWs were due to annealing of the deposited AgNPs previously sputtered on the silicon nanowire. During annealing, small AgNPs would migrate on the nanowire surface and gather to form large AgNPs such that the diameters of the AgNPs ranged from 10 to $120 \mathrm{~nm}$. The high-density AgNP/SiNWs sample exhibits much more
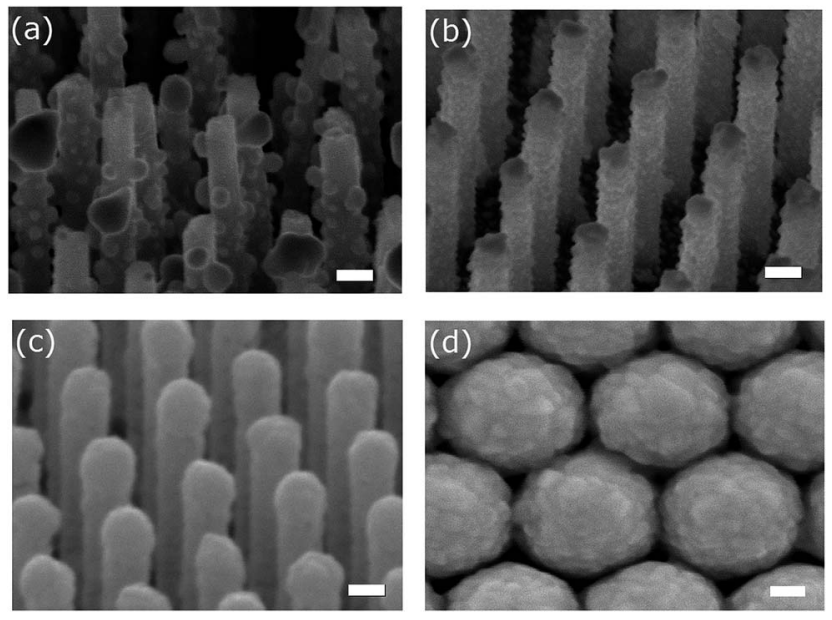

Fig. 1 SEM image of (a) low-density AgNP/SiNWs, fabricated by annealing $\mathrm{Ag}$ layer coated SiNW at $400^{\circ} \mathrm{C}$ for $30 \mathrm{~min}$, (b) high-density AgNP/SiNWs by sputtering Ag for 90 s, (c) Ag/SiNW, and (d) AgFON. All the scale bars indicate $100 \mathrm{~nm}$. uniform AgNPs both in size and distribution on the wire surface than those of the low-density sample. With prolonged sputtering time, the AgNP amount increased and a continuous Ag layer begins to form on the SiNW.

The change from AgNPs to the formation of Ag film on the SiNW is accompanied by evolution of the electric fields. As shown in Fig. 2a and ESI Fig. S1, $\dagger$ the electric field due to localized surface plasmon resonance (LSPR) of the AgNPs is confined on the surface of the nanoparticles and depends on the size when AgNPs are largely separated in low-density AgNP/ SiNWs. When the AgNPs are close to each other as in the case of the high-density AgNP/SiNWs, the field will couple to each other to form gap-based field that depends on both the particle size and the gap spacing between neighboring nanoparticles as shown in Fig. 2b. ${ }^{37-40}$

When the sputtered $\mathrm{Ag}$ forms a continuous film on the SiNWs as the Ag/SiNWs sample, the electric field becomes widely distributed on the wire surface as shown in Fig. 2c. The wide electric field is due to propagating surface plasmon travelling on the Ag surface and depends on the wire diameter (ESI Fig. S2 $\dagger$ ). The wide field free from influence of inter-particle gaps makes Ag/SiNWs a gap-free SERS substrate. ${ }^{9,33}$ Furthermore, due to poor coupling of the illumination light to the localized surface plasmon, the rough $\mathrm{Ag}$ film makes negligible contribution to SERS enhancement as proved in ESI Fig. S3. $\dagger$ Thus, the SERS performance of the Ag/SiNWs substrate depends mainly on the gap-free field on the wire. Another contribution to the field enhancement could be grating effect of two dimensional arrays of metal nanoparticles due to dipolar interaction among the nanoparticles. ${ }^{41,42}$ In the case of $\mathrm{Ag} / \mathrm{SiNWs}$ sample, the inter-wire spacing is about $150 \mathrm{~nm}$ such that the grating effect should be very weak.
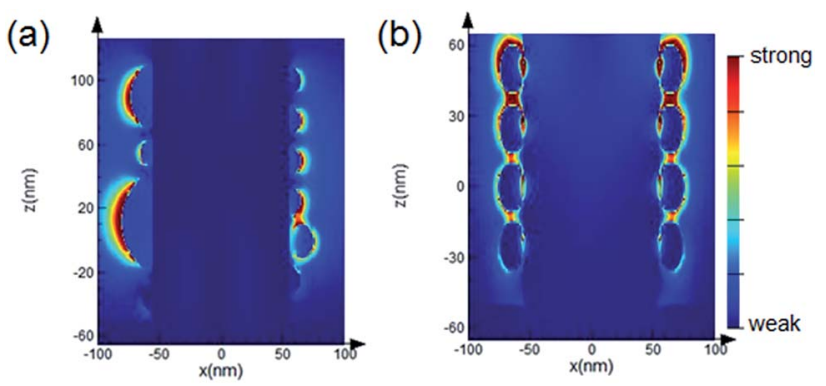

(c)

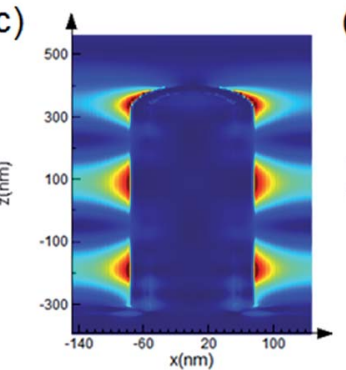

(d)

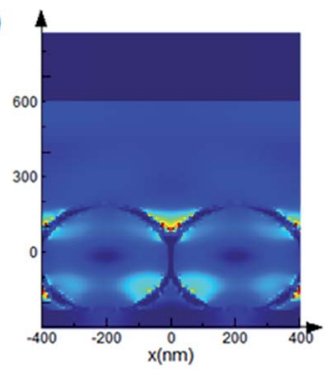

Fig. 2 FDTD-simulated electric fields at surface plasmon resonance: (a) low-density AgNP/SiNWs; (b) high-density AgNP/SiNWs; (c) the gap-free field of Ag/SiNW. (d) The gap-based field of AgFON. The colorbar indicates the field enhancement compared to incident light. 
In contrast, AgFON made by coating a continuous Ag film on a hexagonal-close-packed (HCP) monolayer of uniform nanospheres exhibits a gap-based field that depends mainly on the inter-sphere gaps, as shown in Fig. 2d. Similar to Ag/SiNWs, the roughness of the deposited $\mathrm{Ag}$ film made no contributions to the SERS enhancement. However, the roughness can lead to variation of the inter-sphere gap spacing, significantly change the gap-based field and thus affect the signal reproducibility. Furthermore, the gap-based field is subject to defects of the HCP nanosphere monolayer such as line defect and inconsistent gap distance as shown in ESI Fig. S4. $\dagger$ With the gap-free field from the $\mathrm{Ag} / \mathrm{SiNWs}$ and the gap-based field from the AgFON, we will be able to investigate how the field distribution within the hot spot affects the averaging effect on the reproducibility in the following sections.

The signal reproducibility of a SERS substrate is evaluated by the relative standard deviation (RSD, defined as the standard deviation divided by the mean) of enhancement factors (EFs) of selected peaks of 4-ABT SERS spectra obtained by Raman mapping on a $120 \mu \mathrm{m} \times 120 \mu \mathrm{m}$ area on the SERS substrates. The EFs of the gap-based AgNP/SiNWs is at the scale of $10^{4}$, but the EFs of the gap-based $\mathrm{AgFON}$ and gap-free $\mathrm{Ag} / \mathrm{SiNW}$ are as similar as $7 \times 10^{5}$, as calculated in ESI. $\dagger$ The lower the RSD is, the higher the signal reproducibility the SERS substrate will be. In the following sections, the reproducibility by two averaging effects on the SERS substrate will be evaluated and the mechanism of the reproducibility enhancement by the averaging effects will be analyzed based on the three scales of uniformity: uniform distribution of enhancing nanostructures over a SERS substrate, uniform distribution of the hot spot (or electric field) over all enhancing nanostructures and uniform distribution of analyte molecules over all hot spots.

\section{Increasing hot-spot density}

Firstly, we investigated the averaging effect of increasing hot spot density of a SERS substrate on the reproducibility by comparing the RSDs of 4-ABT molecules adsorbed on the AgNP/ SiNWs with different AgNP densities. The 4-ABT molecules can form a monolayer on $\mathrm{Ag}$ surface even in the AgNP gaps ${ }^{43,44}$ such that the molecule distributions are uniform in all AgNP/SiNWs samples. As shown in Fig. 3a, the RSDs of three signature peaks of 4-ABT SERS spectra (ESI Fig. S5 $\dagger$ ) decrease with increasing density of the AgNPs, achieving less than $20 \%$ for the highdensity AgNP/SiNW sample C. The mapping of $1076 \mathrm{~cm}^{-1}$ Raman peak in Fig. 3c also shows similar trend of signal distribution across the mapping area. The increase of AgNP density actually corresponds to the increased number of the hot spots, which can improve the signal reproducibility and is in agreement with previous reports. ${ }^{31,45}$

The reproducibility improvement by increasing hot spot density in the high-density AgNP/SiNWs should be due to the uniform electric fields of the AgNPs. The AgNPs exhibit more uniform distribution in particle size and spatial distribution on the wire as the density increase from sample A to C in Fig. 3b, which leads to the increased similarity of the AgNP gap-based fields from wire to wire (Fig. 2b). ${ }^{46}$ When the AgNPs are so dense that they form a continuous Ag film on the SiNW as the AgNP/SiNWs sample D, the RSD approaches to that of the Ag/ SiNW and the field is expected to become the gap-free field as in Fig. 2c that is subject only to the nanowire size. Since the SiNWs are uniform in the array, the gap-free field of the AgNP/ SiNW sample D is uniform from wire to wire such that the reproducibility is further increased. The small RSD $(<15 \%)$ of the AgNP/SiNW sample D and the Ag/SiNWs should be due to the gap-free field variation resulted from the $\mathrm{Ag}$ film roughness on the wires.

On the other hand, AgFON exhibits the lower density of hot spots compared to the AgNP/SiNWs substrates. Different from the gap-based field of AgNP/SiNW, the AgFON gap-based field would depend on Ag roughness as well as defects in the nanosphere assembly array, such as non-HCP line defect and inconsistent gap distance as shown in ESI Fig. S4. $\dagger$ Therefore, a low reproducibility of AgFON can be expected. As shown in Fig. 4, the RSDs ( $>40 \%$ ) of the AgFON are more than two times higher than those on the $\mathrm{Ag} / \mathrm{SiNW}$ substrate having the same period of $470 \mathrm{~nm}$. The averaging effect on improving the signal reproducibility in AgFON can reply on using small nanospheres or larger probing areas, as described in the following section.

\section{Influence of probing areas}

Changing objectives of different magnification leads to different sizes of probing spots. The $20 \times$ objective corresponds to a $3 \mu \mathrm{m}$ spot, $50 \times$ objective to a $1.5 \mu \mathrm{m}$ spot and $100 \times$ objective to a $1 \mu \mathrm{m}$ spot. In a SERS measurement, large probing area can cover more hot spots of the substrate but does not necessarily leads to collection of more signals because an objective of low magnification usually has a small numerical aperture or collection angle. The averaging effect of increasing probing area replies on a long-range uniform distribution of the hot spots on the substrate that is not uniform at a short range, ${ }^{31}$ which is useful to overcome the array defects as described below.

Fig. $5 \mathrm{a}$ and $\mathrm{b}$ compare the RSDs of three signature peaks of 4ABT monolayer on the Ag/SiNW and the AgFON substrate with the same period (P) of $470 \mathrm{~nm}$ under different probing areas. The RSDs of $\mathrm{Ag} / \mathrm{SiNW}$ in Fig. 5a decreases by $20 \%$ when the objective changes from the $100 \times$ to the $20 \times$. Since the gap-free field of $\mathrm{Ag} / \mathrm{SiNW}$ depends only on the nanowire size, the RSD drop is due to the suppression of the size variation of the nanowires by probing a $3 \mu \mathrm{m}$ spot of the array. In contrast, the RSD of $4-A B T$ of AgFON drops by $>40 \%$ in Fig. 5b. Significantly, the RSD of $1179 \mathrm{~cm}^{-1}$ peak decreases by almost $100 \%$. The 1179 $\mathrm{cm}^{-1}$ peak was attributed to the transformation of 4-ABT molecules to 4,4'-dimercaptoazobenzene (DMAB) under laser excitation. ${ }^{47}$ The sharp RSD drop of the $1179 \mathrm{~cm}^{-1}$ peak on AgFON could be due to more 4-ABT molecules transformed to DMAB because the $100 \times$ objective delivered much higher excitation energy for the transformation than the $20 \times$ objective. Due to the nature of the AgFON gap-based field, the RSD drops of other two 4-ABT peaks should correspond to the suppression of defects that the HCP nanoparticle array may have in a $1 \mu \mathrm{m}$ spot, including non-uniform sphere size, non-HCP line defect 
(a)

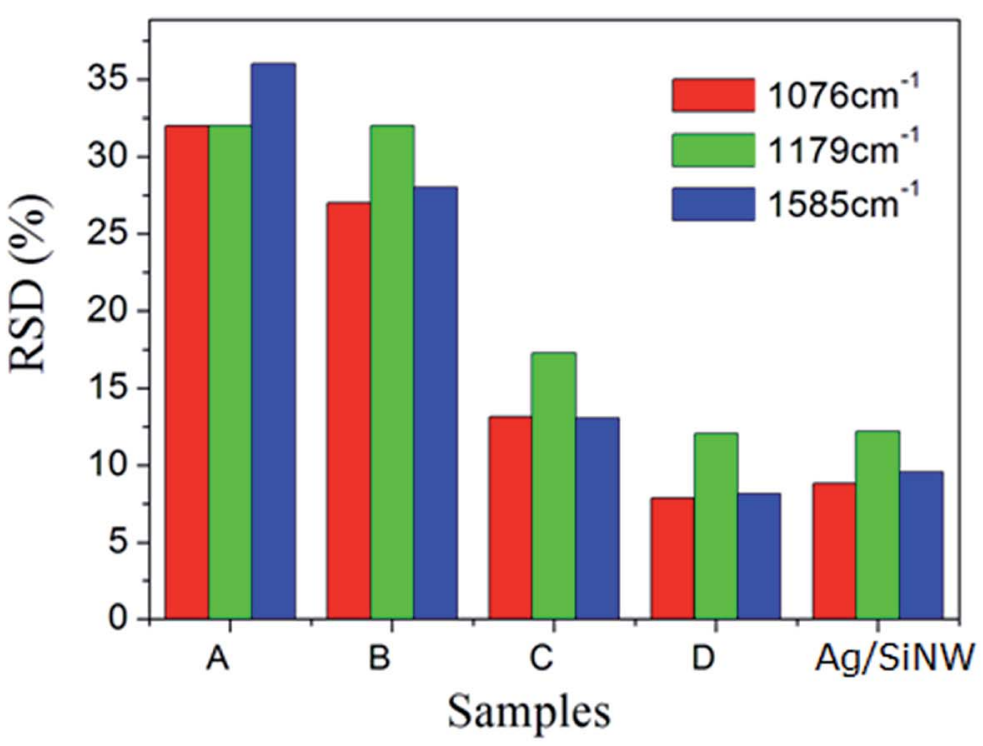

(b)
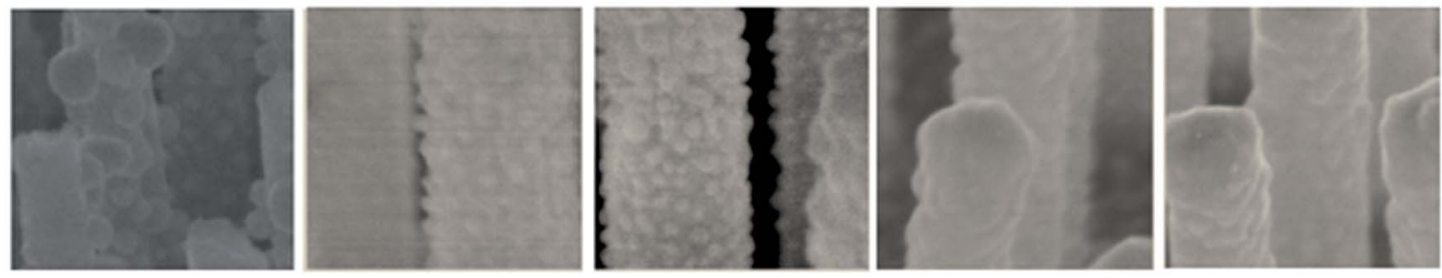

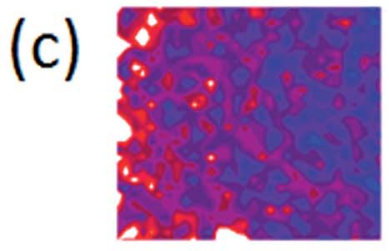

A

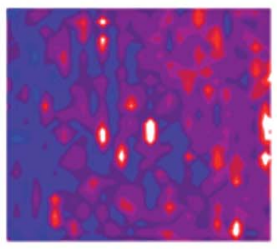

B

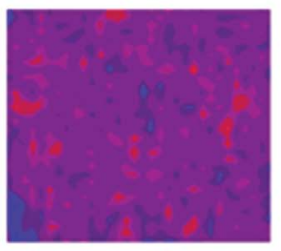

C

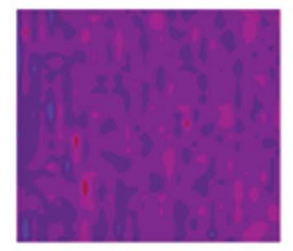

D

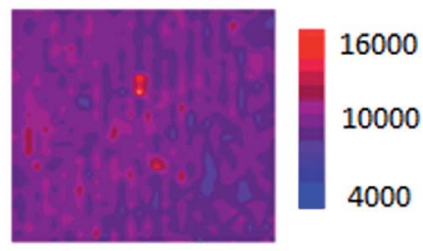

$\mathrm{Ag} / \mathrm{SiNW}$

Fig. 3 (a) RSDs of 4-ABT Raman peaks on AgNP/SiNWs samples with different AgNP densities. (b) Magnified SEM image of AgNP/SiNWs samples $A-D$ and Ag/SiNWs. (c) The colored Raman mapping of $1076 \mathrm{~cm}^{-1}$ peak on $120 \mu \mathrm{m} \times 120 \mu \mathrm{m}$ areas on the samples. The intensity has been normalized. The above silicon nanowires all had periodicities of $300 \mathrm{~nm}$, length around $500 \mathrm{~nm}$ and diameter of $110 \mathrm{~nm}$. Measurement carried out by a $20 \times$ objective.

and inconsistent gap distance. This implies that the array defects that are different in the scale of $1 \mu \mathrm{m}$ become more similar at the scale of $3 \mu \mathrm{m}$.

Similarly, in the Ag/SiNW substrate with a period of $300 \mathrm{~nm}$ in Fig. 5 c, the reproducibility enhancement is also about $20 \%$ by increasing probing area by changing the $100 \times$ objective to the $20 \times$, indicating similar size variation of the nanowires. However, the biggest RSD drop of the AgNP/SiNW of $300 \mathrm{~nm}$ period in Fig. $5 \mathrm{~d}$ is also about $20 \%$, which emphasizes the significant difference between the AgNP gap-based fields and the AgFON gap-based field. The AgNP field depends on the size and gap spacing of AgNPs on an individual nanowire and is not affected by the defects of the nanowire array.

We can summarize how the reproducibility is affected by the substrate uniformity through the electric fields. The reproducibility of the $\mathrm{Ag} / \mathrm{SiNW}$ is the highest because its gap-free field is only affected by the nanowire size variation. AgNP/SiNWs exhibit lower reproducibility because the AgNP gap-based field is affected by AgNP size and the inter-particle gap spacing. The reproducibility of the AgFON is the lowest because the AgFON gap-based field depends on many array defects. The best reproducibility of a SERS substrate corresponds to the field that depends on the least parameters.

It is well-known that the signal reproducibility increases at the cost of SERS enhancement, ${ }^{\mathbf{1 4}}$ and this is the case to the gapbased AgNP/SiNWs and AgFON substrates. For example in Fig. 5, the RSDs of the $1076 \mathrm{~cm}^{-1}$ peak under $100 \times$ objective are $\sim 40 \%$ for the AgNP/SiNWs and $\sim 80 \%$ for the AgFON, respectively, while the corresponding EFs on the two substrates are 2.0 $\times 10^{4}$ and $4.7 \times 10^{5}$. The trade-off relation between reproducibility and enhancement for the gap-based substrates is because smaller nanogaps $(<10 \mathrm{~nm})$ in the hot spots required by higher 


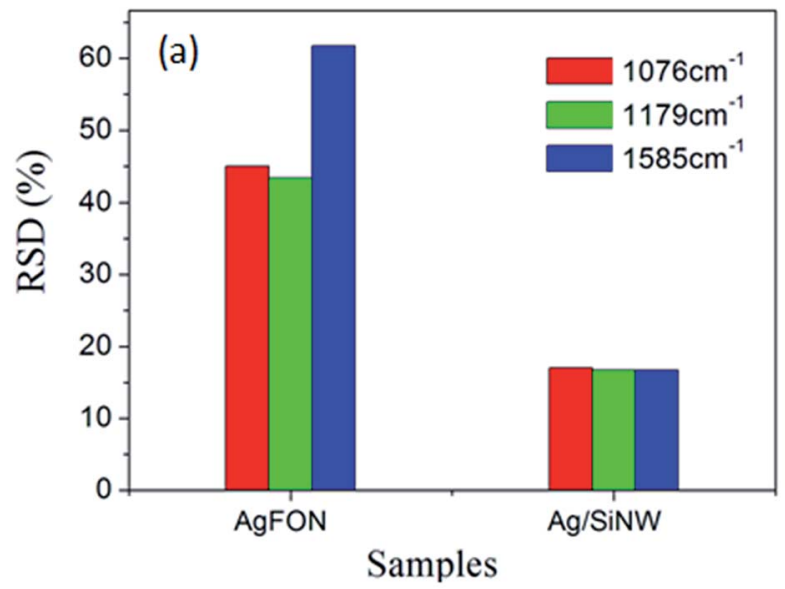

(b)

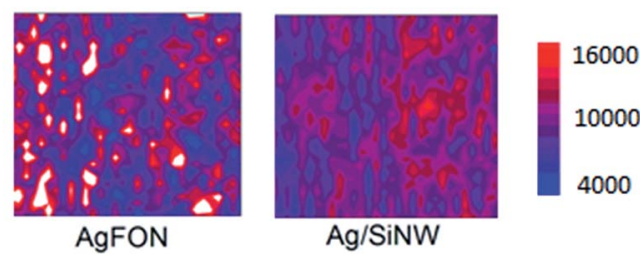

Fig. 4 (a) The RSDs of 4-ABT monolayer on AgFON and Ag/SiNW. (b) The colored Raman mapping of the normalized $1076 \mathrm{~cm}^{-1}$ intensity on $120 \mu \mathrm{m} \times 120 \mu \mathrm{m}$ areas of the corresponding samples.
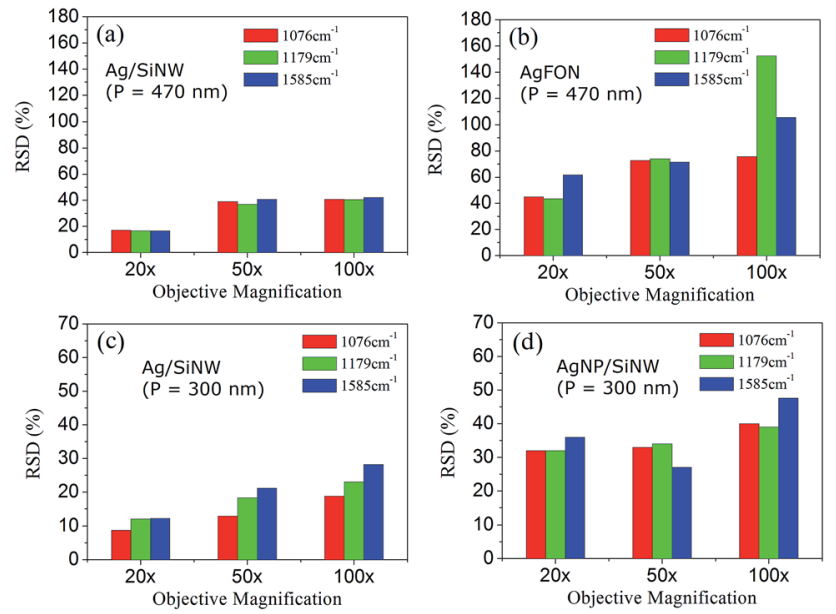

Fig. 5 The influence of objective magnification on the RSD of 4-ABT monolayer on (a) Ag/SiNW with the period as $470 \mathrm{~nm}$, (b) AgFON with the period as $470 \mathrm{~nm}$. (c) $\mathrm{Ag} / \mathrm{SiNW}$ with the period as $300 \mathrm{~nm}$. (d) AgNP/SiNW, with the period as $300 \mathrm{~nm}$.

enhancement can hardly be fabricated uniformly by current nanotechnologies.

However, the trade-off relation does not apply to the gap-free $\mathrm{Ag} / \mathrm{SiNWs}$ substrate. In all the cases we discussed above, the $\mathrm{Ag} /$ SiNWs exhibit higher reproducibility and higher EFs than the AgNP/SiNWs. Similarly, the Ag/SiNWs demonstrate higher reproducibility than the AgFON and their EFs are at the same level. Although AgFON performance can be increased by other fabrication parameters, ${ }^{48}$ the fabrications of the $\mathrm{Ag} / \mathrm{SiNWs}$ and the AgFON used the same parameters to achieve LSPR at $633 \mathrm{~nm}$ wavelength: same nanosphere array as template and same $\mathrm{Ag}$ sputtering time for the continuous Ag layer. It rules out the SERS performance differences being due to different fabrication parameters. The high reproducibility and high enhancement of the $\mathrm{Ag} / \mathrm{SiNWs}$ is actually due to the gap-free electric field as the hot spots: high enhancement requires high density of the gapfree $\Phi 150 \mathrm{~nm}$ nanopillars that can be fabricated uniformly over a large area by nanosphere lithography. Furthermore, the gap-free hot spots allow uniform absorption of analytes while the gap-based hot spots do not, as described in the next section.

\section{Influence of molecule distribution}

A hot spot can contribute to the SERS signal only when an analyte molecule is located inside the spot and excited by the field. Therefore, uniform molecule distribution is the final piece of the reproducibility puzzle that deserves us to investigate. In fact, increasing the number of molecules being probed in a microfluidic SERS detection can improve the SERS reproducibility and is also regarded as a averaging effect. ${ }^{29}$ In the solid-state SERS substrates, increasing uniform molecule distribution in the hot spots can be done by increasing molecule concentration. In the previous sections, the 4-ABT molecule is used to achieve uniform molecule monolayer on the $\mathrm{Ag}$ surface by thiol bonds for the sake of evaluation of the distributions of the hot spots and the electric fields. However in practical SERS applications, most analytes can only physically adsorb on the substrate and their distributions are affected by many parameters, such as the molecule size, conformation and concentration. ${ }^{49,50}$ This section will evaluate the signal reproducibility by using different concentration of a typical SERS molecule, R6G, that physically adsorbs on Ag surface. Fig. 6 show the RSDs of three signature Raman peaks of R6G molecules (ESI Fig. S6 $\dagger$ ) on the three SERS substrates with different periods. The $614 \mathrm{~cm}^{-1}$ peak is associated with $\mathrm{C}-\mathrm{C}-\mathrm{C}$ ring inplane vibration, and 1360 and $1510 \mathrm{~cm}^{-1}$ peaks are assigned to aromatic $\mathrm{C}-\mathrm{C}$ stretching vibrations of the R6G molecule. ${ }^{51}$ The fluctuation as well as the RSD differences of the R6G SERS peaks can be due to different molecular orientations of adsorption and different hot spot geometries though the former might have smaller impact. ${ }^{52,53}$

The RSD difference on the Ag/SiNWs in Fig. 6a decreases with increasing R6G concentration and reaches minimum at $0.02 \mathrm{mM}$, suggesting that R6G monolayer is formed on $\mathrm{Ag}$ surface at $0.02 \mathrm{mM}$ and the peak fluctuation is dominated by hot spot variation. The monolayer formation is supported by calculating the needed amount of R6G molecules for forming a monolayer. In our experiment, a $2 \mu \mathrm{L}$ droplet of R6G solution spreads out as a $3 \mathrm{~mm}$ circle on the SERS substrate. For the $\mathrm{Ag} /$ SiNWs with $700 \mathrm{~nm}$ height, $150 \mathrm{~nm}$ diameter and $470 \mathrm{~nm}$ period, the surface area covered by the $5 \mathrm{~mm}$ circle of the $2 \mu \mathrm{L}$ droplet is $\sim 4.1 \times 10^{13} \mathrm{~nm}^{2}$. One R6G molecule occupies $0.4 \mathrm{~nm}^{2}$ in perpendicular orientation to $\mathrm{Ag}$ surface and $4 \mathrm{~nm}^{2}$ in parallel orientation, respectively. ${ }^{54,55}$ Thus the number of R6G molecules to form monolayer on the $\mathrm{Ag} / \mathrm{SiNW}$ sample is at the scale between $10^{13}$ and $10^{14}$. Therefore, a $2 \mu \mathrm{L}$ droplet of $0.004 \mathrm{mM}$ R6G solution contains $10^{12}$ molecules which are not enough to 

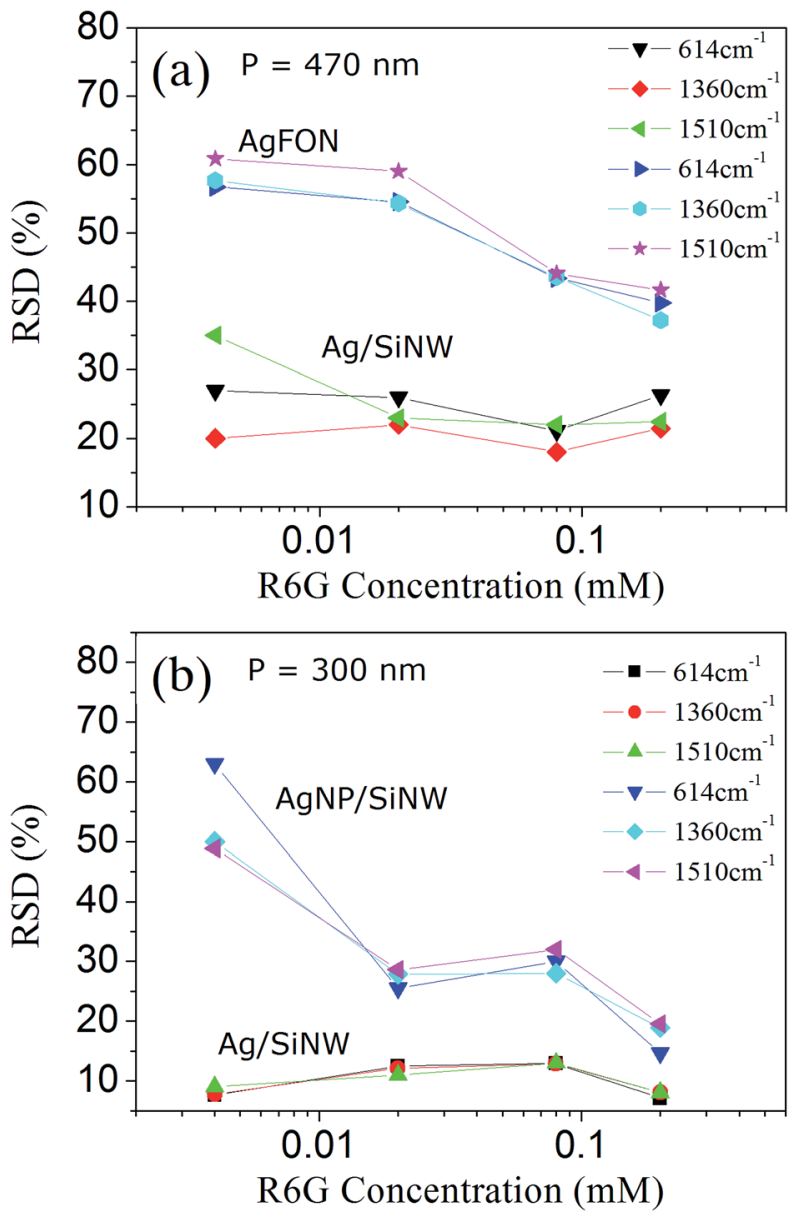

Fig. 6 The influence of R6G concentration on the RSD on (a) AgFON and $\mathrm{Ag} / \mathrm{SiNW}$. The periods are $470 \mathrm{~nm}$. (b) Ag/SiNW and AgNP/SiNW, the periods are $300 \mathrm{~nm}$. The objective used was 20×. The concentrations used are $0.004,0.02,0.08,0.2 \mathrm{mM}$, respectively.

form a monolayer. The obvious RSD difference among the Raman peaks at $4 \times 10^{-6} \mathrm{M}$ in Fig. $6 \mathrm{a}$ is likely due to different molecular orientations of adsorption such that different parts of the molecules experience different electric fields at the hot spots. In contrast, the molecule amount of $2 \mu \mathrm{L} 0.02 \mathrm{mM}$ R6G solution reaches $10^{13}$ that can form monolayer. The RSD difference at $0.02 \mathrm{mM}$ of the $\mathrm{Ag} / \mathrm{SiNWs}$ sample reaches minimum and keep stable in 0.08 and $0.2 \mathrm{mM}$, indicating that all peaks fluctuate similarly depending mainly on the hot spot field distribution. Similar trend can also be observed in the case of the AgNP/SiNWs sample in Fig. 6b and the same assumption of monolayer formation at $0.02 \mathrm{mM}$ applies.

Although the monolayer is formed, the occupations of nanogaps by the R6G molecules are still not uniform and increases with concentrations. As the R6G concentration increases from 0.02 to $0.2 \mathrm{mM}$, the RSDs of the AgFON decrease from $55 \%$ to $40 \%$ in Fig. 6 a. The RSD drop should be due to the increased occupation of the AgFON gaps by the R6G molecules of high concentration. The AgFON gaps are too narrow to allow uniform adsorption of the R6G molecules in them. ${ }^{18}$ As more R6G molecules are adsorbed on the AgFON, the probability of the R6G molecule entering the AgFON gaps increases and thus the occupation of the hot spots becomes more uniform. Similar increased occupation of AgNP gaps also accounts for the $~ 15 \%$ drop of RSDs in the AgNP/SiNWs from concentration at 0.02 to $0.2 \mathrm{mM}$ in Fig. 6b. The $\sim 15 \%$ RSDs of AgNP/SiNWs at $0.2 \mathrm{mM}$ against the high RSD (40\%) of AgFON at the same concentration should be attributed to the averaging effect of increased hot spot density, because the AgNP/SiNWs exhibits much more nanogap hot spots than AgFON.

On the other hand, the RSDs of the gap-free Ag/SiNWs (Fig. 6b) exhibit little changes during the concentration changing, which is due to the gap-free field as well as large wireto-wire separation. The wire-to-wire separation of the Ag/SiNWs is $150 \mathrm{~nm}$ and allows the R6G molecules of low concentration easily adsorb on the wire sidewall and excited by the gap-free field, thus avoiding the occupation problem in the gap-based substrates. In this regard, high R6G concentration will have more R6G molecules adsorb on the wire surface and increase the signal intensity. The molecule distribution in the gap-free field is not changed and thus has little impact to the reproducibility.

\section{Conclusions}

Two approaches leading to averaging effects to improve the SERS signal reproducibility on the gap-based AgNP/SiNWs, AgFON and the gap-free Ag/SiNWs substrates have been evaluated and analysed based on the electric fields of the substrates. The gap-free field of the Ag/SiNWs depends only on the nanowire size. The gap-based fields of AgNP/SiNWs depend on both the AgNP size and the gap spacing. The gap-based field of the AgFON depends on the nanosphere size, the gap spacing as well as nanosphere array defects. With the help of the designed electric fields, the averaging effect of increasing hot spot density in the high-density AgNP/SiNWs is found as a result of the increased similarity of the AgNP fields from wire to wire. The effect of enlarging probing spot on AgFON replies on the longrange uniform distribution of the hot spots. Increasing molecule concentration contributes to the uniform occupation of the gaps in AgFON and AgNP/SiNWs by the analyte molecules. We conclude that the Ag/SiNWs substrate achieves both high reproducibility and high enhancement due to the gap-free electric field that depends on the least parameters. Our finding can be a guide to the design of SERS devices for practical applications.

\section{Acknowledgements}

The authors would like to acknowledge the National Natural Science Foundation of China (No. 51372213).

\section{References}

1 K. E. Shafer-Peltier, C. L. Haynes, M. R. Glucksberg and R. P. Van Duyne, Toward a glucose biosensor based on surface-enhanced Raman scattering, J. Am. Chem. Soc., 2003, 125, 588-593. 
2 D. P. Cowcher, Y. Xu and R. Goodacre, Portable, Quantitative Detection of Bacillus Bacterial Spores Using SurfaceEnhanced Raman Scattering, Anal. Chem., 2013, 85, 32973302.

3 Y. Y. Zhang, Y. Q. Huang, F. L. Zhai, R. Du, Y. D. Liu and K. Q. Lai, Analyses of enrofloxacin, furazolidone and malachite green in fish products with surface-enhanced Raman spectroscopy, Food Chem., 2012, 135, 845-850.

4 S. E. J. Bell, J. N. Mackle and N. M. S. Sirimuthu, Quantitative surface-enhanced Raman spectroscopy of dipicolinic acid towards rapid anthrax endospore detection, Analyst, 2005, 130, 545-549.

5 X. X. Han, G. G. Huang, B. Zhao and Y. Ozaki, Label-Free Highly Sensitive Detection of Proteins in Aqueous Solutions Using Surface-Enhanced Raman Scattering, Anal. Chem., 2009, 81, 3329-3333.

6 K. N. Heck, B. G. Janesko, G. E. Scuseria, N. J. Halas and M. S. Wong, Observing Metal-Catalyzed Chemical Reactions In Situ Using Surface-Enhanced Raman Spectroscopy on Pd-Au Nanoshells, J. Am. Chem. Soc., 2008, 130, 16592-16600.

7 J. M. Yuen, N. C. Shah, J. T. Walsh, M. R. Glucksberg and R. P. Van Duyne, Transcutaneous Glucose Sensing by Surface-Enhanced Spatially Offset Raman Spectroscopy in a Rat Model, Anal. Chem., 2010, 82, 8382-8385.

8 Y. Zhao, Y.-L. Zhang, J.-A. Huang, Z. Zhang, X. Chen and W. Zhang, Plasmonic nanopillar array embedded microfluidic chips: an in situ SERS monitoring platform, $J$. Mater. Chem. A, 2015, 3(12), 6408-6413.

9 Y. Zhao, J.-A. Huang, Z. Zhang, X. Chen and W. Zhang, Quantitative analysis of multiplex-components and double stranded DNA by wide-range surface-enhanced Raman spectroscopy based on ordered $\mathrm{Ag} / \mathrm{Si}$ nanowire arrays, $J$. Mater. Chem. A, 2014, 2(26), 10218-10224.

10 S. K. Yang, X. M. Dai, B. B. Stogin and T. S. Wong, Ultrasensitive surface-enhanced Raman scattering detection in common fluids, Proc. Natl. Acad. Sci. U. S. A., 2016, 113(2), 268-273.

11 M. J. Banholzer, J. E. Millstone, L. D. Qin and C. A. Mirkin, Rationally designed nanostructures for surface-enhanced Raman spectroscopy, Chem. Soc. Rev., 2008, 37, 885-897.

12 E. J. Blackie, E. C. Le Ru and P. G. Etchegoin, SingleMolecule Surface-Enhanced Raman Spectroscopy of Nonresonant Molecules, J. Am. Chem. Soc., 2009, 131, 14466-14472.

13 K. Kneipp, Y. Wang, H. Kneipp, L. T. Perelman, I. Itzkan, R. Dasari and M. S. Feld, Single molecule detection using surface-enhanced Raman scattering (SERS), Phys. Rev. Lett., 1997, 78, 1667-1670.

14 M. J. Natan, Concluding remarks - Surface enhanced Raman scattering, Faraday Discuss., 2006, 132, 321-328.

15 S. L. Kleinman, R. R. Frontiera, A. I. Henry, J. A. Dieringer and R. P. Van Duyne, Creating, characterizing, and controlling chemistry with SERS hot spots, Phys. Chem. Chem. Phys., 2013, 15(1), 21-36.
16 J. R. Lombardi and R. L. Birke, A Unified View of SurfaceEnhanced Raman Scattering, Acc. Chem. Res., 2009, 42(6), 734-742.

17 S. Lal, N. K. Grady, J. Kundu, C. S. Levin, J. B. Lassiter and N. J. Halas, Tailoring plasmonic substrates for surface enhanced spectroscopies, Chem. Soc. Rev., 2008, 37(5), 898911.

18 Y. Fang, N.-H. Seong and D. D. Dlott, Measurement of the distribution of site enhancements in surface-enhanced Raman scattering, Science, 2008, 321(5887), 388-392.

19 K. A. Willets, S. M. Stranahan and M. L. Weber, Shedding Light on Surface-Enhanced Raman Scattering Hot Spots through Single-Molecule Super-Resolution Imaging, J. Phys. Chem. Lett., 2012, 3(10), 1286-1294.

20 A. Lombardi, A. Demetriadou, L. Weller, P. Andrae, F. Benz, R. Chikkaraddy, J. Aizpurua and J. J. Baumberg, Anomalous Spectral Shift of Near- and Far-Field Plasmonic Resonances in Nanogaps, ACS Photonics, 2016, 3(3), 471-477.

21 E. C. Le Ru, E. Blackie, M. Meyer and P. G. Etchegoin, Surface enhanced Raman scattering enhancement factors: a comprehensive study, J. Phys. Chem. C, 2007, 111, 1379413803.

22 S. De Vitis, M. L. Coluccio, F. Gentile, N. Malara, G. Perozziello, E. Dattola, P. Candeloro and E. Di Fabrizio, Surface enhanced Raman spectroscopy measurements of MCF7 cells adhesion in confined micro-environments, Optics and Lasers in Engineering, 2016, 76, 9-16.

23 A. Sujith, T. Itoh, H. Abe, K. I. Yoshida, M. S. Kiran, V. Biju and M. Ishikawa, Imaging the cell wall of living single yeast cells using surface-enhanced Raman spectroscopy, Anal. Bioanal. Chem., 2009, 394(7), 1803-1809.

24 J. P. Camden, J. A. Dieringer, J. Zhao and R. P. Van Duyne, Controlled Plasmonic Nanostructures for SurfaceEnhanced Spectroscopy and Sensing, Acc. Chem. Res., 2008, 41(12), 1653-1661.

25 M. Moskovits, Surface-enhanced Raman spectroscopy: a brief retrospective, J. Raman Spectrosc., 2005, 36(6-7), 485-496.

26 E. C. Le Ru, J. Grand, I. Sow, W. R. C. Somerville, P. G. Etchegoin, M. Treguer-Delapierre, G. Charron, N. Felidj, G. Levi and J. Aubard, A Scheme for Detecting Every Single Target Molecule with Surface-Enhanced Raman Spectroscopy, Nano Lett., 2011, 11(11), 5013-5019.

27 H. Wang, C. S. Levin and N. J. Halas, Nanosphere arrays with controlled sub-10-nm gaps as surface-enhanced Raman spectroscopy substrates, J. Am. Chem. Soc., 2005, 127, 14992-14993.

28 H. Wang, C. S. Levin and N. J. Halas, Nanosphere arrays with controlled sub-10-nm gaps as surface-enhanced Raman spectroscopy substrates, J. Am. Chem. Soc., 2005, 127(43), 14992-14993.

29 L. X. Chen and J. B. Choo, Recent advances in surfaceenhanced Raman scattering detection technology for microfluidic chips, Electrophoresis, 2008, 29(9), 1815-1828.

30 E. Smith and G. Dent, Modern Raman spectroscopy: a practical approach, J. Wiley, Hoboken, NJ, 2005, p. 210. 
31 M. S. Schmidt, J. Hubner and A. Boisen, Large Area Fabrication of Leaning Silicon Nanopillars for Surface Enhanced Raman Spectroscopy, Adv. Mater., 2012, 24(10), OP11-OP18.

32 A. Barhoumi and N. J. Halas, Label-Free Detection of DNA Hybridization Using Surface Enhanced Raman Spectroscopy, J. Am. Chem. Soc., 2010, 132, 12792-12793.

33 J. A. Huang, Y. Q. Zhao, X. J. Zhang, L. F. He, T. L. Wong, Y. S. Chui, W. J. Zhang and S. T. Lee, Ordered Ag/Si Nanowires Array: Wide-Range Surface-Enhanced Raman Spectroscopy for Reproducible Biomolecule Detection, Nano Lett., 2013, 13(11), 5039-5045.

34 J. A. Huang, Y. Q. Zhao, X. J. Zhang, L. B. Luo, Y. K. Liu, J. A. Zapien, C. Surya and S. T. Lee, Enhanced Raman scattering from vertical silicon nanowires array, Appl. Phys. Lett., 2011, 98(18), 183108.

35 K. Q. Peng, X. Wang, L. Li, X. L. Wu and S. T. Lee, Highperformance silicon nanohole solar cells, J. Am. Chem. Soc., 2010, 132(20), 6872-6873.

36 Z. P. Huang, N. Geyer, P. Werner, J. de Boor and U. Gosele, Metal-Assisted Chemical Etching of Silicon: A Review, $A d v$. Mater., 2011, 23(2), 285-308.

37 E. Petryayeva and U. J. Krull, Localized surface plasmon resonance: Nanostructures, bioassays and biosensing-A review, Anal. Chim. Acta, 2011, 706(1), 8-24.

38 M. Ringler, T. A. Klar, A. Schwemer, A. S. Susha, J. Stehr, G. Raschke, S. Funk, M. Borowski, A. Nichtl, K. Kurzinger, R. T. Phillips and J. Feldmann, Moving nanoparticles with Raman scattering, Nano Lett., 2007, 7(9), 2753-2757.

39 W. D. Li, F. Ding, J. Hu and S. Y. Chou, Three-dimensional cavity nanoantenna coupled plasmonic nanodots for ultrahigh and uniform surface-enhanced Raman scattering over large area, Opt. Express, 2011, 19(5), 3925-3936.

40 J. D. Caldwell, O. Glembocki, F. J. Bezares, N. D. Bassim, R. W. Rendell, M. Feygelson, M. Ukaegbu, R. Kasica, L. Shirey and C. Hosten, Plasmonic Nanopillar Arrays for Large-Area, High-Enhancement Surface-Enhanced Raman Scattering Sensors, ACS Nano, 2011, 5(5), 4046-4055.

41 L. P. Du, X. J. Zhang, T. Mei and X. C. Yuan, Localized surface plasmons, surface plasmon polaritons, and their coupling in 2D metallic array for SERS, Opt. Express, 2010, 18(3), 19591965.

42 Y. Z. Chu, E. Schonbrun, T. Yang and K. B. Crozier, Experimental observation of narrow surface plasmon resonances in gold nanoparticle arrays, Appl. Phys. Lett., 2008, 93(18), 1108.

43 N. Mohri, S. Matsushita, M. Inoue and K. Yoshikawa, Desorption of 4-aminobenzenethiol bound to a gold surface, Langmuir, 1998, 14(9), 2343-2347.
44 J. B. Jackson, S. L. Westcott, L. R. Hirsch, J. L. West and N. J. Halas, Controlling the surface enhanced Raman effect via the nanoshell geometry, Appl. Phys. Lett., 2003, 82(2), 257-259.

45 Y. J. Oh and K. H. Jeong, Glass Nanopillar Arrays with Nanogap-Rich Silver Nanoislands for Highly Intense Surface Enhanced Raman Scattering, Adv. Mater., 2012, 24(17), 2234-2237.

46 X.-Y. Liu, J.-A. Huang, B. Yang, X.-J. Zhang and Y.-Y. Zhu, Highly reproducible SERS substrate based on polarizationfree $\mathrm{Ag}$ nanoparticles decorated $\mathrm{SiO}_{2} / \mathrm{Si}$ core-shell nanowires array, AIP Adv., 2015, 5(5), 057159.

47 Y. F. Huang, H. P. Zhu, G. K. Liu, D. Y. Wu, B. Ren and Z. Q. Tian, When the Signal is not from the Original Molecule to be Detected: Chemical Transformation of para-Aminothiophenol on $\mathrm{Ag}$ during the SERS Measurement, J. Am. Chem. Soc., 2010, 132(27), 9244-9246.

48 N. G. Greeneltch, M. G. Blaber, A. I. Henry, G. C. Schatz and R. P. Van Duyne, Immobilized Nanorod Assemblies: Fabrication and Understanding of Large Area SurfaceEnhanced Raman Spectroscopy Substrates, Anal. Chem., 2013, 85(4), 2297-2303.

49 L. A. Gearheart, H. J. Ploehn and C. J. Murphy, Oligonucleotide adsorption to gold nanoparticles: A surface-enhanced Raman spectroscopy study of intrinsically bent DNA, J. Phys. Chem. B, 2001, 105(50), 12609-12615.

50 W. H. Han, S. M. Lindsay, M. Dlakic and R. E. Harington, Kinked DNA, Nature, 1997, 386(6625), 563.

51 D. Pristinski, S. L. Tan, M. Erol, H. Du and S. Sukhishvili, In situ SERS study of rhodamine 6G adsorbed on individually immobilized Ag nanoparticles, J. Raman Spectrosc., 2006, 37(7), 762-770.

52 J. A. Dieringer, A. D. McFarland, N. C. Shah, D. A. Stuart, A. V. Whitney, C. R. Yonzon, M. A. Young, X. Y. Zhang and R. P. Van Duyne, Surface enhanced Raman spectroscopy: new materials, concepts, characterization tools, and applications, Faraday Discuss., 2006, 132, 9-26.

53 A. A. Moore, M. L. Jacobson, N. Belabas, K. L. Rowlen and D. M. Jonas, 2D correlation analysis of the continuum in single molecule surface enhanced Raman spectroscopy, $J$. Am. Chem. Soc., 2005, 127(20), 7292-7293.

54 A. Kudelski, Raman studies of rhodamine 6G and crystal violet sub-monolayers on electrochemically roughened silver substrates: Do dye molecules adsorb preferentially on highly SERS-active sites?, Chem. Phys. Lett., 2005, 414(46), 271-275.

55 R. Sasai, T. Fujita, N. Iyi, H. Itoh and K. Takagi, Aggregated structures of rhodamine $6 \mathrm{G}$ intercalated in a fluortaeniolite thin film, Langmuir, 2002, 18(17), 6578-6583. 